\title{
Técnicas quirúrgicas para la preservación de esfínter en cáncer de recto bajo: revisión histórica y estado actual
}

\author{
Katya Carrillo G. ${ }^{1}$, Mario Abedrapo M. ${ }^{2}$ y Rodrigo Azolas M. ${ }^{2}$
}

'Residente de Investigación en Coloproctología, Departamento de Cirugía Hospital Clínico Universidad de Chile. Santiago, Chile.

¿Unidad de Coloproctología

Departamento de Cirugía,

Hospital Clínico Universidad

de Chile. Santiago, Chile.

Recibido el 8 de julio de 2017 y aceptado para publicación el

7 de agosto de 2017

Correspondencia a: Dr. Mario Abedrapo M. mabedrapo@hcuch.cl

\section{Surgical techiniques for sphincter preservation y low rectal cancer: historical review and current status}

After multiple attempts to achieve appropriate results in the surgical resection for rectal cancer, Sir William Ernest Miles develops the technique that would carry his name, and is, without modifications, currently referred as abdominoperineal resection. This technique, considered gold standard for rectal tumors for many years, has been gradually replaced by sphincter preserving surgery. Low anterior resection allowed sphincter-preservation in patients with low rectal tumors within $5 \mathrm{~cm}$ from the anal verge. Technical developments (double stapling technique), better understanding of oncological principles (total mesorectal excision) and the introduction of neoadyuvant treatment with chemoradiotherapy, allowed further lowering of the adequate distal resection margin and to carry out ultralow colorectal and coloanal anastomoses. Ultralow intersphincteric resection removes the internal anal sphincter (partial, subtotal or total resection) avoiding permanent colostomy in a subset of patients. This technique has been broadly studied with published oncological outcomes that are better or similar to those of abdominoperineal resection. Currently, evaluation of response after neoadyuvant therapy has led some working groups to propose organ preservation; either by strict follow up for complete clinical response, or by local excision techniques for incomplete clinical response.

Key words: rectal cancer; surgery; sphincter-preservation.

\section{Resumen}

Tras múltiples intentos para lograr resultados apropiados en una resección quirúrgica por cáncer de recto, Sir William Ernest Miles desarrolla la técnica que posteriormente llevará su nombre, y que se conoce actualmente, sin muchas modificaciones, como resección abdominoperineal. Esta técnica, considerada el gold standard para tumores de recto por muchos años, fue progresivamente reemplazada por las técnicas de preservación de esfínter. La resección anterior baja permitió preservar el esfínter a paciente con tumores de recto a más de $5 \mathrm{~cm}$ del margen anal. El desarrollo técnico (suturas grapadas), mejor entendimiento de principios oncológicos (resección total del mesorrecto) y la introducción de la neoadyuvancia con quimioradioterapia, permitió bajar el margen de sección distal considerado adecuado y realizar anastomosis colorrectales ultrabajas y coloanales. La resección ultrabaja interesfintérica reseca el esfínter anal interno (en forma total, subtotal o parcial) logrando evitar la colostomía permanente en un grupo de pacientes. Esta técnica, ha sido ampliamente estudiada con resultados oncológicos publicados que resultan mejores o similares que los de la resección abdominoperineal. Actualmente la evaluación de respuesta a neoadyuvancia, ha llevado a algunos grupos de trabajo a plantear la preservación del órgano; ya sea mediante el seguimiento estricto tras respuesta clínica completa, como técnicas de resección local en respuesta incompleta. Palabras clave: cáncer rectal; cirugía; preservación de esfínter.

\section{Introducción histórica}

Tras los intentos fallidos de algunos cirujanos del siglo XVIII, la verdadera cirugía en cáncer de recto comienza en 1826, cuando Jacques Lisfranc realiza la primera resección exitosa de recto por vía perineal. Desde entonces, otras resecciones similares fueron realizadas, entre ellos, la casuística de Theodore Billroth. El acceso posterior (descrito inicialmente por Verneuil y Kocher) fue popularizado por 
Paul Kraske tras presentar su técnica en el Congreso de la Sociedad Alemana de Cirujanos en $1885^{1}$. Ésta incluía la resección del ala izquierda del sacro y cóccix para lograr un mejor acceso para la posterior resección del tumor y anastomosis primaria. Una revisión de 1.500 casos operados durante el siglo XIX, demostró una mortalidad perioperatoria de $21 \%$, y, debido a que no existían nociones de una resección oncológicamente adecuada, la tasa de recurrencia local era altísima, cercana al $80 \%{ }^{1}$.

Paralelamente Vincent Czerny realiza la primera cirugía con abordaje abdominal y perineal en 1884 , técnica posteriormente perfeccionada y descrita en 1908 por Sir William Ernest Miles. Particularmente frustrado por la alta recurrencia local, Miles, enfatizó en la diseminación por vía linfática principalmente ascendente, pero también descendente y lateral, lo que lo llevó a proponer una resección en block más anatómicamente correcta. La operación de Miles describía los siguientes principios: a) El "ano abdominal" (colostomía terminal) es una necesidad; b) el colon "pélvico" debe ser resecado ya que su irrigación está contenida en el área de diseminación ascendente; c) todo el "mesocolon pélvico" debe ser resecado con una pestaña de peritoneo bajo la bifurcación de las arterias iliacas; d) los linfonodos sobre la bifurcación de las iliacas deben ser removidos; y por último e) la resección perineal debe ser lo más amplia posible ${ }^{2}$. Con estos principios logró reducir la recidiva local de un $80 \%$ a un $29,5 \%$, lo cual fue muy significativo, sin embargo, el costo fue un aumento de la mortalidad perioperatoria inicial de hasta un $42 \%$, hecho por el cual fue ampliamente criticado $^{2}$. Los avances en el cuidado perioperatorio, anestesia, transfusiones y otros mejorarían esta cifra sustancialmente, convirtiendo a la resección abdominoperineal (RAP) en el gold standard de tratamiento del cáncer de recto bajo.

\section{Cirugía con preservación de esfínter: Primeras experiencias y estado actual}

Posteriores estudios, especialmente por el patólogo inglés Cuthbert Dukes, con respecto a la diseminación linfática que restaban importancia a la diseminación descendente en tumores rectales más proximales, permitieron a Donald Balfour en 1910 realizar la primera resección anterior baja (RAB) con anastomosis colorrectal. Tras perfeccionar la técnica, Claude Dixon, logra reducir la mortalidad y mejorar los resultados oncológicos adecuados ${ }^{3}$. Esto marca el inicio de la cirugía en cáncer de recto con preservación de esfínter, sin embargo, para los tumores a $5 \mathrm{~cm}$ del margen anal la RAP se mantuvo como la técnica de elección.

Dificultades técnicas propias de la pelvis hacían compleja las anastomosis bajas ya que hasta el momento la técnica se llevaba a cabo manualmente con suturas en "paracaídas" para tumores a más de $6 \mathrm{~cm}$ del margen anal, o bien por vía anal para anastomosis más bajas, según lo descrito por Sir Alan Parks ${ }^{4,5}$. En 1977 se realiza la primera sutura mecánica con grapadora circular, permitiendo realizar técnicamente de forma segura anastomosis más bajas ${ }^{6}$.

Dos elementos fundamentales colaboran en la mayoría de resultados de la cirugía con preservación de esfínter y disminuir el margen necesario: la resección total del mesorrecto (TME) y el uso de terapia neoadyuvante. La TME, descrita por R.J. Heald en 1982, marca un hito en la cirugía por cáncer de recto con una considerable disminución en la recurrencia local, siendo una técnica reproducible, se transformó rápidamente en un estándar de tratamiento, disminuyendo considerablemente los pacientes que se sometían a una $\mathrm{RAP}^{7,8}$. El uso de la radioterapia para el cáncer de recto se inició a principio del siglo XX, pero fue en la década de los noventa que se inició como un estándar de tratamiento. Sauer et al. en el 2004, publica los beneficios del uso de radioquimioterapia neoadyuvante por sobre la adyuvante, demostrando mejor adherencia, menor tasa de efectos adversos, además de una reducción significativa de un $50-70 \%$ en la tasa de recurrencia local y menor estenosis de anastomosis 9 . Actualmente, el uso de radioterapia acortada preoperatoria no ha demostrado inferioridad en sus resultados oncológicos. El esquema más utilizado incluye cirugía dentro de los siguientes 7-10 días, sin embargo, un estudio reciente ha demostrado mejores resultados en cuanto a complicaciones posoperatorias con una espera mayor de entre 4 a 8 semanas ${ }^{10}$.

Fue en los años 80-90 que el margen distal de $5 \mathrm{~cm}$, considerado oncológicamente adecuado, fue sustituido por $2 \mathrm{~cm}$ tras los estudios de anatomía patológica posoperatoria inicialmente publicados ${ }^{11}$. Sin embargo, posteriormente aparecen nuevos estudios en pacientes con y sin neoadyuvancia en donde márgenes distales $<1 \mathrm{~cm}$ no provocaban diferencias en la sobrevida global, ni sobrevida libre de enfermedad $^{12}$. Actualmente, las guías de la National Comprehensive Cancer Network (NCCN) Guideli$n e s^{\circledR}$ aceptan 1-2 cm como margen adecuado ${ }^{13}$.

El abordaje inicialmente abierto, ha sido sustituido progresivamente por el abordaje laparoscópico. En cirugía de cáncer de colon, la laparoscopía es, sin duda, actualmente el método de elección; sin embargo, en cirugía por cáncer de recto esto no ha sido 
tan ampliamente aceptado. Los estudios actuales no son concluyentes en términos de los resultados oncológicos, pero sí han demostrado los beneficios quirúrgicos a corto plazo asociados al abordaje laparoscópico ${ }^{14}$. La cirugía robótica se ha posicionado actualmente, promulgando beneficios teóricos, sin embargo, la evidencia aún no avala este abordaje como la primera elección.

Aunque el margen distal considerado oncológicamente adecuado ha disminuido, la dificultad técnica de una pelvis estrecha, pacientes obesos y tumores voluminosos en la disección del recto y mesorrecto distal al tumor, se ha asociado con mayor tasa de conversión, mayor tasa de perforación y de margen circunferencial positivo, incluso en manos expertas. Lo anterior representa, en ciertos casos, una limitante a la preservación de esfínter. El abordaje transanal con dispositivos e instrumental similar al de la laparoscopia (Figura 1) para la TME, tiene el beneficio teórico de entregar una evaluación directa del margen distal, mejor visualización, y por lo tanto, lograr una mejor calidad del mesorrecto. Fue el mismo R.J. Heald quien la describió como una técnica revolucionaria, sin embargo, la evidencia no ha logrado demostrar un beneficio franco por sobre las otras técnicas ${ }^{15}$. A nivel nacional, las series de

Figura 1. Puerto y disección transanal (GelPoint Path ${ }^{\circledR}$. casos publicados han demostrado buenos resultados quirúrgicos ${ }^{16}$. La cirugía robótica, también ha abordado el problema de la pelvis estrecha con la ventaja que ofrecen sus instrumentos con mayor grado de libertad de movimiento, sin embargo, esto tampoco se ha traducido en un beneficio sustancial ${ }^{17}$. El estudio ROLARR, que busca comparar una serie de datos quirúrgicos, oncológicos y funcionales, aún no publica sus resultados definitivos.

En la actualidad, la RAB y la resección anterior ultrabaja (RAUB, con anastomosis a menos de 6 $\mathrm{cm}$ del margen anal), representan el tratamiento quirúrgico de elección en los pacientes con tumores a más de $2 \mathrm{~cm}$ del margen anal. Esto no sólo por la posibilidad técnica y evitar la colostomía definitiva, sino también porque la mayoría de la evidencia actual muestra menor tasa de complicaciones y mejores resultados oncológicos: menor tasa de borde circunferencial positivo, menor tasa de recurrencia local y mejor sobrevida a 5 años ${ }^{18}$. En cuanto a resultados funcionales y su impacto en la calidad de vida, los resultados son variables. Sabemos que hasta un $90 \%$ de estos pacientes presentará un cambio en el hábito intestinal ${ }^{19}$. Existe una entidad descrita llamada "Síndrome de resección anterior baja" (LARS) de etiología multifactorial, que se caracteriza por síntomas como incontinencia, urgencia, aumento de la frecuencia y fragmentación de las deposiciones ${ }^{20}$. A pesar que, hay estudios que demuestran una mejoría hasta 12 meses posquirúrgico $^{21}$, hasta un $41 \%$ de los pacientes presenta LARS en grado mayor ${ }^{22}$ con una importante alteración de la calidad de vida $^{23}$ (cuantificado mediante una encuesta breve validada en varios idiomas, incluido el español chileno ${ }^{24}$.

\section{Resección ultrabaja interesfintérica (RAUBIE)}

Con el fin de lograr resecciones más bajas, pero conservando la función del esfínter evitando así la colostomía definitiva, nace la RAUBIE. Descrita por Schiessel ${ }^{25}$ en 1994, incluye la resección parcial $(1 / 3)$, subtotal $(2 / 3)$ o total de esfínter anal interno (incisión a nivel de la línea dentada, entre línea dentada y surco interesfintérico y a nivel del surco interesfintérico, respectivamente) asistida con retractores (i.e Lone Star $^{\circledR}$ ) (Figura 2) con una disección a través del plano interesfintérico (Figura 3) y efectuando la TME ya sea por vía abdominal o transanal. Algunos grupos quirúrgicos han realizado variaciones, como por ejemplo, realizar además resección parcial del esfínter anal externo. La reconstitución la 
continuidad intestinal se realiza mediante el descenso del colon distal sin tensión, con o sin liberación del ángulo esplénico, con o sin construcción de un reservorio ("pouch") y sutura coloanal manual e ileostomía en asa de protección (Figura 3).

Desde su introducción, la RAUBIE ha cobrado mayor importancia, disminuyendo considerablemente la tasa de resecciones abdominoperineales que se realizan para tumores de recto a $<5 \mathrm{~cm}$ del margen anal. Las indicaciones de esta técnica son razonablemente uniformes en la literatura internacional. Tumores T1-3, son los candidatos a RAUBIE, con o sin neoadyuvancia; por el contrario, tumores T4, fijos a la pelvis, con invasión del esfínter anal externo, mal diferenciados, enfermedad metastásica incurable, mala función esfinteriana preoperatoria y estado mental alterado, son contraindicaciones para esta técnica.

En cuanto a resultados quirúrgicos, se describe una tasa variable de morbilidad asociada, entre un $7,8 \%$ y $38,5 \%$, con una mortalidad perioperatoria entre $0 \%$ y $1,7 \%$.

La filtración de la anastomosis ocurre entre un 5-10\% ( $\sin$ embargo, hay reportes de hasta 48\%), y estenosis entre un $8,4 \%$ y $15,9 \%$. Abscesos pélvicos y necrosis del colon descendido también son complicaciones descritas cercanas a un 5 y $2 \%$, respectivamente. La tasa de reoperaciones fluctúa entre el $0 \%$ y $12,9 \%$. Existe hasta un $10 \%$ de pacientes que acaban con ostomía definitiva, ya sea por imposibilidad de cerrar la ileostomía, como por incontinencia, recidiva o complicaciones que requieran la confección de un ostoma definitivo ${ }^{26}$.

Los resultados oncológicos de la literatura reportados se resumen en la Tabla 1 . Al menos tres autores $^{26}$ realizan comparación en cuanto a recurrencia local y sobrevida entre la RAUBIE y la RAP, sin diferencias significativas entre ellas, a excepción de la sobrevida global a 5 años en el artículo de Saito et al. que muestra beneficio de la RAUBIE por sobre la RAP27.

En cuanto a resultados funcionales, no existe un estándar para medir las alteraciones funcionales y el grado de incontinencia en la literatura y cómo se afecta la calidad de vida. El grado de incontinencia medido mediante Score de Wexner o Cleveland Clinic Fecal Incontinence Score (CCFIS) ha mostrado mejorar con el tiempo, con un promedio inicial de 17 puntos a los 3 meses y disminuye a 7,8 puntos a los 24 meses. El sexo masculino y el uso de neoadyuvancia se consideran entre posibles factores de riesgo para una peor función, así como el cierre de ileostomía precoz (dentro de los 6 primeros meses) se ha considerado protector en

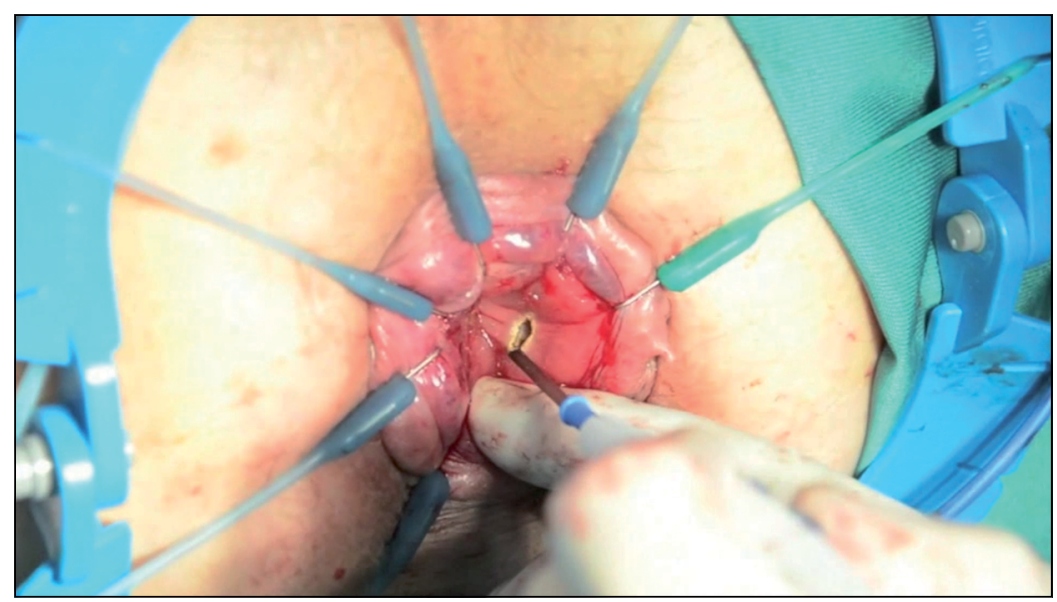

Figura 2. Incisión interesfintérica con retractor (LoneStar $\left.{ }^{\circledR}\right)$.

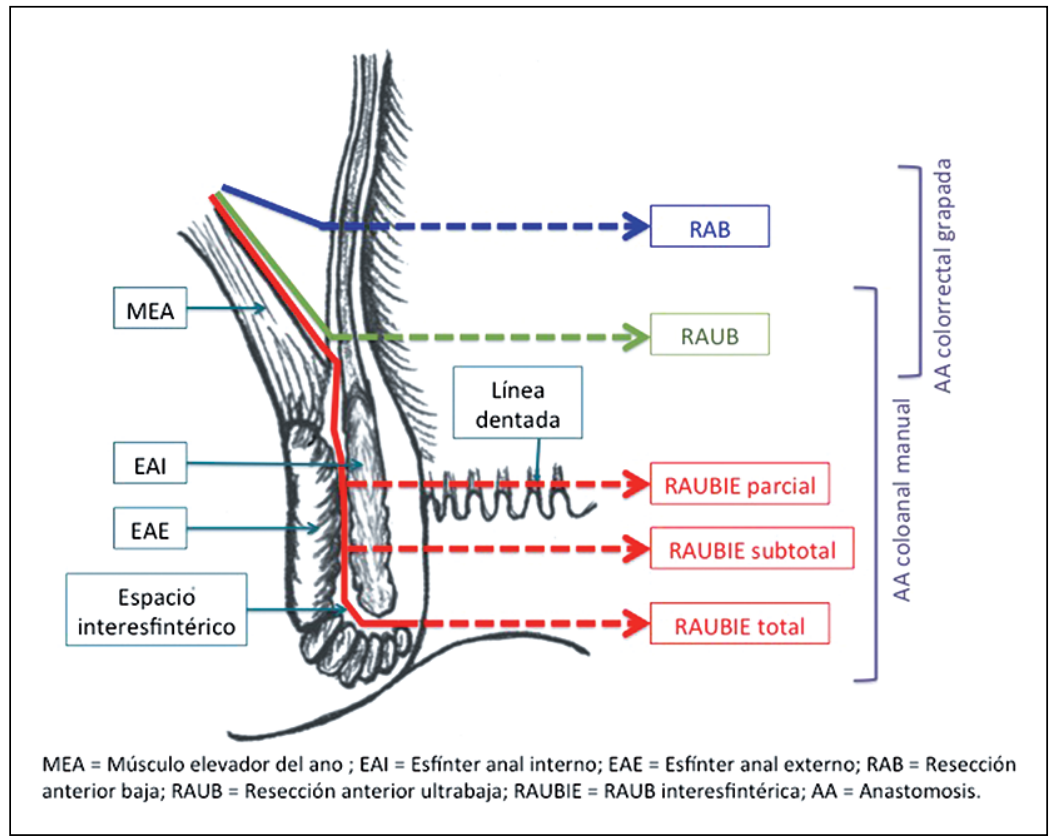

Figura 3. Técnicas quirúrgicas para la preservación de esfínter. Adaptado de Akagi, $2013^{26}$.

Tabla 1. Resultados oncológicos de RAUBIE. Resumen de datos publicados

\begin{tabular}{|ll|}
\hline Ítem oncológico & \\
\hline Tasa R0 & $92-100 \%$ \\
\hline Margen circunferencial $\leq 1 \mathrm{~mm}$ & $4-13,3 \%$ \\
\hline Recurrencia global & $13,3-19,4 \%$ \\
\hline Recurrencia a distancia & $2,5-19 \%$ \\
\hline Recurrencia local & $0-22,7 \%$ \\
\hline Sobrevida global a 5 años & $79-97 \%$ \\
\hline Sobrevida libre de enfermedad a 5 años & $69-86 \%$ \\
\hline
\end{tabular}


algunos estudios ${ }^{27-29}$. En globalidad, se reportan resultados satisfactorios con adecuada continencia de hasta $68 \%$ de los pacientes, sin embargo, aún existe un grupo de pacientes que deben revertir la reconstitución para quedar con colostomía definitiva por mala función ${ }^{27,28}$. La confección de un reservorio colónico en $\mathrm{J}$ ha logrado algunos beneficios en esta área ${ }^{30,31}$. La RAUBIE tiene peores resultados funcionales que el resto de las cirugías con preservación de esfínter ${ }^{32}$, sin embargo, y a pesar de una alta tasa de incontinencia en algunos estudios, la calidad de vida es mejor que la asociada a la RAP 33 .

\section{Técnicas para preservar el órgano: Resección local y "Watch and Wait"}

El tratamiento neoadyuvante con quimioradioterapia ha determinado que, en ciertos grupos de pacientes, el paradigma del tratamiento quirúrgico radical sea reevaluado por terapias más conservadoras, dependiendo de la respuesta y grado regresión tumoral. La cirugía tradicional tiene una serie de desventajas, como las complicaciones posoperatorias propias del procedimiento, la posibilidad de ostomía definitiva, alteraciones en la función urinaria, defecatoria y sexual, causando un impacto en la calidad de vida de los pacientes.

Existe un porcentaje cercano al $25 \%$ (incluso algunos reportan hasta un $42 \%$ ) de pacientes en los cuales se logra una respuesta patológica completa (RPC), evaluado en piezas operatorias de pacientes operados en que la biopsia diferida no logró identificar restos de células tumorales ${ }^{34,35}$. Se discute, actualmente, si estos pacientes son sometidos a una cirugía innecesaria, con los riesgos y complicaciones que esta conlleva. Es por esto que, emergen nuevas alternativas de tratamiento para este grupo de pacientes con respuesta a la neoadyuvancia, como lo son el "watch-and-wait" (manejo conservador sin cirugía y seguimiento estricto) y la resección local transanal posterior a la neoadyuvancia, mediante resección convencional o cirugía mínimamente invasiva (TEMS, TAMIS).

El desafío está determinado en identificar a qué pacientes se les puede ofrecer estos tratamientos más conservadores. Para esto se requiere identificar a quienes presentan una respuesta clínica completa (RCC), que corresponde a la determinación, mediante una serie de elementos de evaluación clínica, que no existe tumor residual a pesar de no contar con la confirmación histopatológica. La evaluación parte con el tacto rectal y la evaluación endoscó- pica ya sea rígida o flexible. Si bien no forman parte de la evaluación para definir una RCC, el uso de técnicas de imágenes complementarias resulta indispensable en la actualidad, especialmente en la evaluación del mesorrecto. La resonancia magnética, especialmente utilizando protocolo de difusión, permite actualmente evaluar el mesorrecto y determinar, con una buena concordancia/correlación con la biopsia, el grado de regresión tumoral, pudiendo incluso identificar mejor a los pacientes con RPC. El uso de PET-CT también ha demostrado en algunos estudios relacionarse con la regresión tumoral, sin embargo, faltan estudios y su uso de rutina no está recomendado ${ }^{35}$.

En caso de RCC, los grupos que promueven el "watch and wait" en estos casos, han reportado recurrencia local de $31 \%$ a 5 años de seguimiento, la que suele ser precoz (dentro de los primeros 12 meses) y que la cirugía de salvataje es posible en el 93\% de los pacientes con resultados oncológicos similares a los pacientes con respuesta incompleta operados según protocolo estándar ${ }^{35,36}$. El uso de boost de radioterapia o el uso de quimioterapia "de consolidación" para mejorar los resultados de esta estrategia están aún en estudio.

En casos de respuesta incompleta, (ypT1-2) la resección local muestra resultados menos que satisfactorios, con alta tasa de recurrencia local y tasas de complicación de la herida de un 25 a $70 \%{ }^{35}$. La preservación del órgano en estos pacientes debe ser cuidadosamente evaluada, ya que no existe evidencia consistente que la avale.

La resección local sin uso de neoadyuvancia está reservada para tumores $\mathrm{T} 1$ pequeños $(<3 \mathrm{~cm})$ y de histología favorable (bien diferenciados, sin invasión linfovacular, etc. $)^{13}$. El uso de esta técnica en cT1 que no cumplan los criterios anteriores, o cT2 está reservada para pacientes de alto riesgo quirúrgico o que rechacen la cirugía, con resultados bastante limitados ${ }^{37}$.

\section{Discusión}

Se ha recorrido un largo camino en el desarrollo de técnica que permitan preservar el esfínter. El manejo multimodal y multidisciplinario del cáncer de recto ha permitido el desglose de pacientes en subgrupos, que permiten un manejo diferencial y en los cuales es posible evitar la ostomía definitiva. Actualmente, la mayoría de los pacientes con cáncer de recto bajo, logran preservar el esfínter con resultados oncológicos adecuados y una alteración variable de la función y calidad de vida. 


\section{Responsabilidades éticas}

Protección de personas y animales. Los autores declaran que para esta investigación no se han realizado experimentos en seres humanos ni en animales.

Confidencialidad de los datos. Los autores declaran que en este artículo no aparecen datos de pacientes.

Derecho a la privacidad y consentimiento informado. Los autores declaran que en este artículo no aparecen datos de pacientes.

\section{Financiación}

Los autores declaran no haber recibido ninguna financiación para la realización de este trabajo.

\section{Conflicto de intereses}

Los autores declaran no tener ningún conflicto de intereses.

\section{Bibliografía}

1. Graney MJ, Graney CM. Colorectal surgery from antiquity to the modern era. Dis Colon Rectum 1980;23:432-41.

2. Miles WE. A method of performing abdomino-perineal excision for carcinoma of the rectum and of the terminal portion of the pelvic colon (1908). CA Cancer J Clin. 1971;21:361-4.

3. Dixon CF. Anterior Resection for Malignant Lesions of the Upper Part of the Rectum and Lower Part of the Sigmoid. Ann Surg. 1948;128:425-42.

4. Ruo L, Guillem JG. Major 20th-century advancements in the management of rectal cancer. Dis Colon Rectum 1999;42:56378

5. Fleshman JW, Smallwood N. Current concepts in rectal cancer. Clin Colon Rectal Surg. 2015;28:5-11.

6. Moran BJ. Stapling instruments for intestinal anastomosis in colorectal surgery. Br J Surg. 1996;83:902-9.

7. Heald RJ, Husband EM, Ryall RD. The mesorectum in rectal cancer surgery: the clue to pelvic recurrence? Br J Surg. 1982;69:613-6.

8. Heald RJ, Moran BJ, Ryall RD, Sexton $\mathrm{R}$, MacFarlane JK. Rectal cancer: the Basingstoke experience of total mesorectal excision, 1978-1997. Arch Surg. 1998;133:894-9.

9. Sauer R, Becker H, Hohenberger W, Rödel C, Wittekind C, Fietkau R,et al German Rectal Cancer Study Group. Preoperative versus postoperative chemoradiotherapy for rectal cancer. $\mathrm{N}$ Engl J Med. 2004;351:1731-40.

10. Erlandsson J, Holm T, Pettersson D, Berglund Å, Cedermark B, Radu C, et al. A. Optimal fractionation of preoperative radiotherapy and timing to surgery for rectal cancer (Stockholm III): a multicentre, randomised, non-blinded, phase 3, non-inferiority trial. Lancet Oncol. 2017;18:336-46.

11. Pollett WG, Nicholls RJ. The relationship between the extent of distal clearance and survival and local recurrence rates after curative anterior resection for carcinoma of the rectum. Ann Surg. 1983;198:15963.

12. Mukkai Krishnamurty D, Wise PE. Importance of surgical margins in rectal cancer. J Surg Oncol. 2016;113:323-32.

13. NCCN Clinical Practice Guidelines in Oncology (NCCN Guidelines ${ }^{\circledR}$ ). Rectal Cancer. Version 3.2017-March 13, 2017. https://www.nccn.org/professionals/ physician_gls/PDF/rectal.pdf

14. Atallah C, Efron JE. Laparoscopy for Rectal Cancer. Clin Colon Rectal Surg. 2017;30:104-11.

15. Hasegawa S, Takahashi R, Hida K, Kawada K, Sakai Y. Transanal total mesorectal excision for rectal cancer. Surg Today 2016;46:641-53.

16. Abedrapo M, López S, Azolas R, Díaz M, Sanguineti A, Llanos JL, et al. Resección total del mesorrecto por vía transanal en cáncer de recto: Análisis de resultados de una serie preliminar en un hospital universitario. Rev Chil Cir. 2017;69:53-9.

17. Xiong B, Ma L, Zhang C, Cheng Y. Robotic versus laparoscopic total mesorectal excision for rectal cancer: a meta-analysis. J Surg Res. 2014;188:40414.

18. Wang XT, Li DG, Li L, Kong FB, Pang LM, Mai W. Meta-analysis of oncological outcome after abdominoperineal resection or low anterior resection for lower rectal cancer. Pathol Oncol Res. 2015;21:19-27.
19. Bryant CL, Lunniss PJ, Knowles CH, Thaha MA, Chan CL. Anterior resection syndrome. Lancet Oncol. 2012;13:e403-8.

20. Williamson ME, Lewis WG, Holdsworth PJ, Finan PJ, Johnston D. Decrease in the anorectal pressure gradient after low anterior resection of the rectum. A study using continuous ambulatory manometry. Dis Colon Rectum 1994;37:1228-31.

21. Emmertsen KJ, Laurberg S; Rectal Cancer Function Study Group. Impact of bowel dysfunction on quality of life after sphincter-preserving resection for rectal cancer. Br J Surg. 2013;100:1377-87.

22. Bregendhal S, Emmertsen KJ, Lous J, Laurberg S. Bowel dysfunction after low anterior resection with and without neoadjuvant therapy for rectal cancer: a population based cross-sectional study. Colorectal Dis 2013;15:1130-9.

23. Juul T, Ahlberg M, Biondo S, Espin E, Jiménez LM, Matzel KE, et al. Low anterior resection syndrome and quality of life: an international multicenter study. Dis Colon Rectum 2014;57:585-91.

24. López S, Carrillo K, Sanguineti A, Azolas R, Díaz M, Bocic G, et al . Adaptación transcultural del cuestionario acerca de la función intestinal (LARS Score) para su aplicación en pacientes operados de cáncer de recto medio y bajo. Rev Chil Cir. 2017; 69:44-8

25. Schiessel R, Karner-Hanusch J, Herbst F, Teleky B, Wunderlich M. Intersphincteric resection for low rectal tumours. Br J Surg. 1994;81:1376-8.

26. Akagi Y, Kinugasa T, Shirouzu K. Intersphincteric resection for very low rectal cancer: a systematic review. Surg Today 2013;43:838-47.

27. Saito N, Sugito M, Ito M, Kobayashi A, Nishizawa Y, Yoneyama Y, et al 


\section{ARTíCULO DE REVISIÓN}

Oncologic outcome of intersphincteric resection for very low rectal cancer. World J Surg. 2009;33:1750-6.

28. Saito N, Ito M, Kobayashi A, Nishizawa Y, Kojima M, Nishizawa Y, et al. Longterm outcomes after intersphincteric resection for low-lying rectal cancer. Ann Surg Oncol. 2014;21:3608-15.

29. Hughes DL, Cornish J, Morris C. LARRIS Trial Management Group. Functional outcome following rectal surgerypredisposing factors for low anterior resection syndrome. Int J Colorectal Dis. 2017;32:691-7.

30. Heriot AG, Tekkis PP, Constantinides V, Paraskevas P, Nicholls RJ, Darzi A, et al. Meta-analysis of colonic reservoirs versus straight coloanal anastomosis after anterior resection. Br J Surg. 2006;93: 19-32.
31. Park JG, Lee MR, Lim SB, Hong CW, Yoon SN, Kang SB, et al. Colonic J-pouch anal anastomosis after ultralow anterior resection with upper sphincter excision for low-lying rectal cancer. World J Gastroenterol. 2005;11: 2570-3.

32. Konanz J, Herrle F, Weiss C, Post S, Kienle P. Quality of life of patients after low anterior, intersphincteric, and abdominoperineal resection for rectal cancer--a matched-pair analysis. Int $\mathrm{J}$ Colorectal Dis. 2013;28:679-88.

33. Klose J, Tarantino I, Kulu Y, Bruckner T, Trefz S, Schmidt T, et al. SphincterPreserving Surgery for Low Rectal Cancer: Do We Overshoot the Mark? J Gastrointest Surg. 2017;21:885-91.

34. Fichera A, Allaix ME. Paradigm-shifting new evidence for treatment of rectal cancer. J Gastrointest Surg. 2014;18: 391-7.

35. São Julião GP, Habr-Gama A, Vailati BB, Araujo SEA, Fernández LM, et al. New Strategies in Rectal Cancer. Surg Clin North Am. 2017;97:587-604.

36. Habr-Gama A, Gama-Rodrigues J, São Julião GP, Proscurshim I, Sabbagh C, Lynn PB, Pérez RO. Local recurrence after complete clinical response and watch and wait in rectal cancer after neoadjuvant chemoradiation: impact of salvage therapy on local disease control. Int J Radiat Oncol Biol Phys. 2014;88:822-8.

37. Heafner TA, Glasgow SC. A critical review of the role of local excision in the treatment of early ( $\mathrm{T} 1$ and $\mathrm{T} 2$ ) rectal tumors. J Gastrointest Oncol. 2014;5:34552. 